\title{
RESISTOR NETWORK AS MODELING TOOL FOR FRACTURE DETECTION IN CRYSTALLINE ROCKS
}

\author{
Jaroslav JIRK ${ }^{1,2)}$ *and Jan VILHELM ${ }^{1)}$ \\ 1) Charles University, Faculty of Science, Institute of Hydrogeology, Engineering Geology and Applied Geophysics, \\ Albertov 6, Prague, Czech Republic \\ 2) G IMPULS Praha spol. s r.o., Pristavni 24, Prague, Czech Republic
}

*Corresponding author's e-mail: jirku4@natur.cuni.cz

\section{ARTICLE INFO}

Article history:

Received 13 September 2019

Accepted 5 November 2019

Available online 13 November 2019

\section{Keywords:}

ERT

Resistor network

Forward problem

Res2Dmod

Geophysics

Resistivity

\begin{abstract}
The rock massif with sparse fracture network can be considered as highly inhomogeneous. The fractures enable electric current to flow in otherwise relatively non-conductive country rock. The measured apparent resistivity in such a medium strongly depends on the mutual position of the individual electrodes in relation to the conductive fractures. Commonly used 2D resistivity models are built up by rectangular blocks with finite dimensions and characterized by their resistivity values. The fractures are however very thin compared to the model block size. Electrical resistivity of fractures can be in this case modelled only by reduction of resistivity value in the individual blocks containing fractures. We have developed a resistor-network model where the medium is replaced by discrete $2 \mathrm{D}$ orthogonal resistors lattice. Instead of resistivity blocks this model is built up by individual resistors which are characterized by their resistance value. The proposed resistor network model can be used for evaluating the electric resistivity measurements in underground storage monitoring projects for the interpretation of the ERT measurements carried out directly on the surface of crystalline rocks with individual fractures. The model can be used for modelling the artificial conductors as well. The effect of fractures and fracture zones is presented together with an example from real measurement in an underground tunnel.
\end{abstract}

\section{INTRODUCTION}

The rock massif with sparse fracture network can be considered as highly inhomogeneous medium from the point of view of its electrical resistivity properties. The fractures enable electric current to flow in otherwise relatively non-conductive country rock. Fractures can be very thin and highly conductive. These fractures can be of an irregular shape and they can be mutually interconnected. The measured apparent resistivity in such a medium strongly depends on the mutual position of the individual electrodes in relation to the conductive fractures. This is especially pronounced in the case of resistivity measurement performed directly on the rock surface with fractures.

Commonly used 2D resistivity models are made of rectangular blocks with finite dimensions. The individual blocks are characterized by their resistivity values. The size of the blocks is usually increasing with increasing depth which corresponds to decreasing value of the so-called sensitivity function (Loke, 2019; Butler, 2017). The fractures are however very thin compared to the model block size. Electrical resistivity of fractures can be in this case modelled only by reduction of the resistivity value in the individual blocks containing fractures. The uncertainty of the fracture position and the resistivity distribution is significantly smoothed and blurred then.

After first initial attempts (Vilhelm and Jirků, 2018) we developed a resistor-network model to solve this issue. In this case the medium is replaced by a discrete $2 \mathrm{D}$ orthogonal resistors lattice. Instead of resistivity value $[\Omega \mathrm{m}]$ which represents continuous material property this model is built up by individual resistors which are characterized by their resistance values [ $\Omega]$.

Resistor network model is used for evaluating apparent resistivity pseudosections in the ERT method. We demonstrate the possibilities of this model for Wenner-alpha and Wenner-Schlumberger electrode arrays. Some basic model calculations demonstrate agreement between the finite-element calculation (Res2DMod by Loke) and proposed resistor network model. Performed model calculations demonstrate also the new possibility for modelling of anisotropic resistivity medium. The resistor network model is also suitable for modelling thin artificial conductors' effect such as metallic wells, anchors, pipes or rebars (Rucker, 2012; Ronczka et al., 2015; Dahlin and Loke, 2018). 


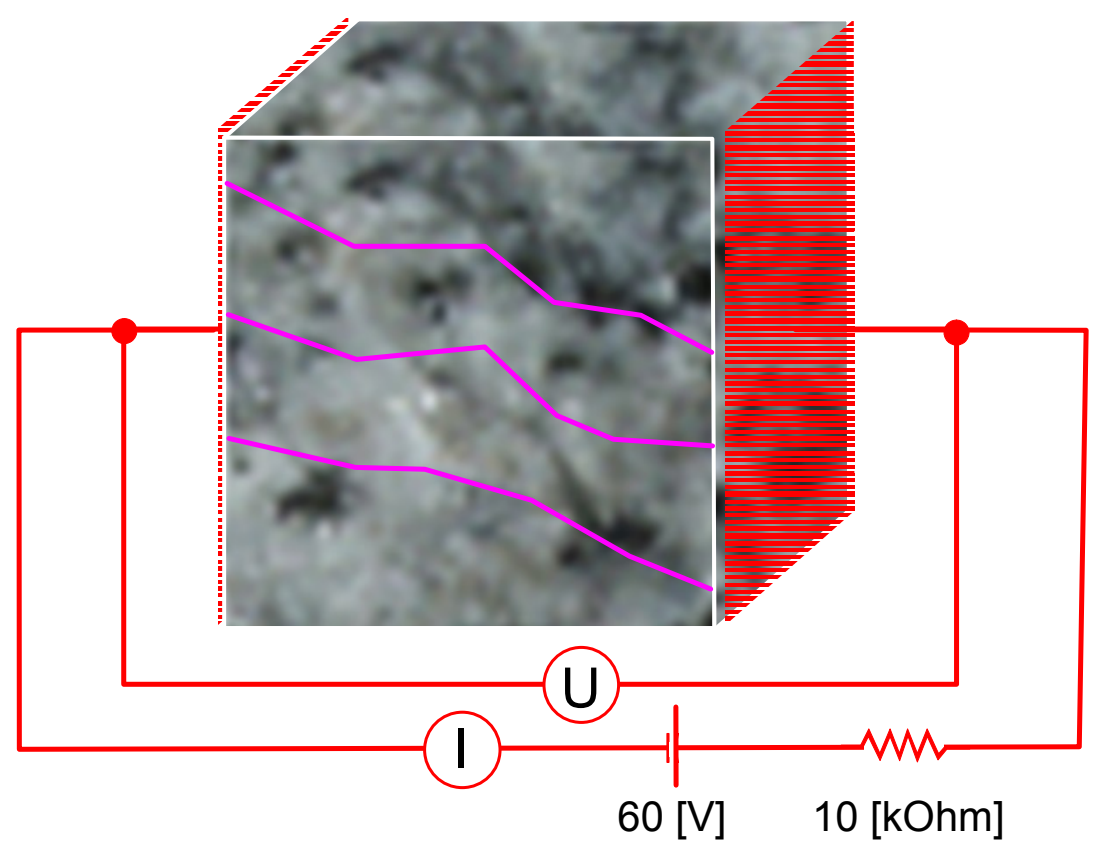

Fig. 1 The array of the resistance laboratory tests on the rock samples.

The proposed resistor network model can be for instance used for evaluation of the electric resistivity measurements in the underground storage monitoring projects for the interpretation of the ERT measurements carried out directly on the crystalline rocks surface with individual fractures. Such a conclusion was confirmed by an extensive study of relation between joints' frequency and hydraulic permeability in the Czech massif granites down to depths of 300 meters (Rukavičková, 2008). The authors found out that granites show the indirect relation between number of joints and permeability, i.e. the connection seems to be more or less random. The direct relation (being crucial for considering granites a geo-barrier) occurs in case of sparse but interconnected fractures. The resistor network model seems to be a tool for founding out the presence of an interconnected fracture in a form of thin localized area that can change electrical conductivity according to the saturation swiftly and strongly.

\section{FRACTURES IN CRYSTALLINE ROCKS}

Disruption of unweathered and compact crystalline rocks by fractures is very often of a local character, even in case of a disrupted zone presence. In a rock massif one can observe separate fractures. The electrical resistivity of such otherwise nonconductive rocks then strongly depends on the fractures' presence in a close vicinity of a place where the resistivity is measured and also on the fractures water saturation together with the joint water's mineralization. This can be documented on an experiment we carried out - we measured the disrupted (by fractures) rock samples' resistance in the lab conditions. The samples had a cubical shape of $8 \mathrm{~cm}$-long sides. Two stainless metallic electrodes were attached on the pairs of opposite specimen sidewalls - Figure 1. The size of the electrodes was the same as size of the sample sidewall. To ensure electrical connection of the electrodes with the specimen a highly conductive contact medium based on water suspension of $\mathrm{CuSO}_{4} \cdot 5 \mathrm{H}_{2} \mathrm{O}$ mixed with flour was used. The rock sample was connected in series with the $10 \mathrm{k} \Omega$ resistor and DC stabilized electrical source of $60 \mathrm{~V}$. Using precise Hewlett-Packard 3466A micro-voltmeter a voltage drop on the sample was measured. Together with the known value of electric current in the circuit it is possible to calculate resistance of the sample by means of the Ohm's law. The sample resistance can be transformed into material's electrical resistivity using the known crosssection area of a rock specimen.

The electric resistivity measurements on the dry samples and on the samples with different levels of water saturation were then performed. The volumetric weight and open porosity were estimated according to the modified ČSN EN 1936 (2007) standard. Open porosity is defined by mutually connected systems of pores that are water-permeable and allow the flow, socalled effective porosity, and tiny non-connected pores and microcracks on a sample's surface, which can be saturated with water (Norton and Knapp, 1977).

The electrical resistance was always measured in three perpendicular directions on each cubical sample. First, the samples were completely saturated by mineralized water (conductivity of approximately $150 \mu \mathrm{S} / \mathrm{cm}$ ). Samples saturation took 24 hours and it was carried out at ambient temperature and under low pressure conditions using vacuum pump. Then the samples were slightly dried out to two decreased saturation levels and their electrical resistance was remeasured. Finally, the samples were completely driedout during the temperature of $105{ }^{\circ} \mathrm{C}$ for 24 hours. 


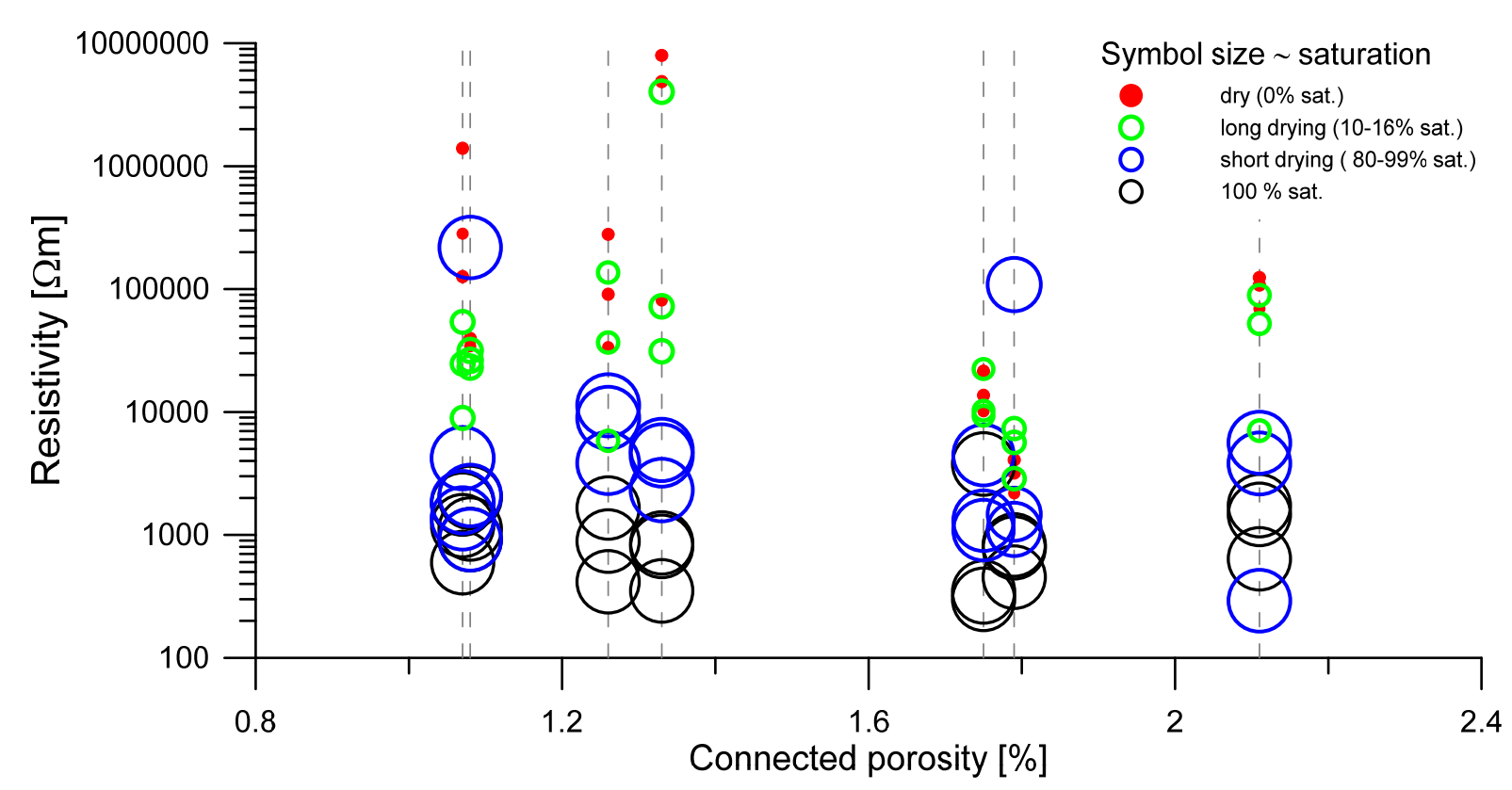

Fig. 2 Resistivity of a series of samples with different connected porosity at different saturation levels. Cubic specimens, resistivity values measured in three different directions for each specimen.

Heating up the samples to desired temperature was gradual with exponentially increasing temperature from $20^{\circ} \mathrm{C}$ for approximately 4 hours (about one degree per three minutes). The sense of such gradual heating is preventing from new microcracks generation due to high thermal gradient during the process (according to Zeng et al. (2019) and Reuschle et al. (2006)).

Measured resistance values of all samples in all directions are displayed in Figure 2. Separate samples are characterized by their connected porosity. For each sample all three values of resistance are displayed (i.e. three orthogonal directions). Colors distinguish different saturation levels - $100 \%$ saturation, short and long drying and $0 \%$ saturation eventually. The saturation level in case of partly saturated samples is expressed by the symbol size and varies for separate samples. The meaning of Figure 2 graph is proving the fact that crystalline rocks disrupted by fractures can change the resistance in several orders due to connected porosity and different saturation. The places with disruptions represent a strong inhomogeneity of a rock environment but such an inhomogeneity can be very thin and local at the same time. Its influence is strongly based on the presence of a physical contact with measuring electrode. Due to position between the electrode and fracture the value of apparent resistivity may change rapidly.

\section{RESISTOR NETWORK MODEL}

The resistor network model is an alternative to commonly used 2D model that is based on division the medium into the blocks of a constant apparent resistivity (Fig .3).
Model using the blocks of preset value of resistivity is suitable mainly in cases of relatively homogenous environment or when it does not contain thin bodies of significantly different resistivity. While using blocks sizes in range of meters during modelling environment with fractures or thin fracture zones of decimeters a significant blurring of the resistivity model occurs, in terms of geometry and the resistivity values as well. Contrary to this the model based on the resistor network allows natural implementation of a thin conductive structure that is characterized mainly by its high conductivity and length (its width does not have instant meaning). The calculation on the resistor network model is displayed in Figure 4.

The calculation of the resistor network model is based on the Kirchhoff laws. In particular it is based on calculation of all loop currents, marked in Figure 4. The calculation leads to the linear equations system. According to calculated loop currents one can get the voltage between any two points on the surface, i.e. to model a resistance measurement via arbitrary electrodes array. It is also possible to use the loop currents themselves to characterize the current distribution in an environment which shows for instance the influence of a single model's parts to the final apparent resistivity.

Contrary to the resistivity distribution model the resistor model does not represent a real physical environment's model but, while used suitably, it can be used for the model calculation of apparent resistivity distribution. The essence is mainly in fact that the real physical property is the material resistivity while the value of resistance representing an electronic component does not say anything about its physical dimensions. It is known that during 

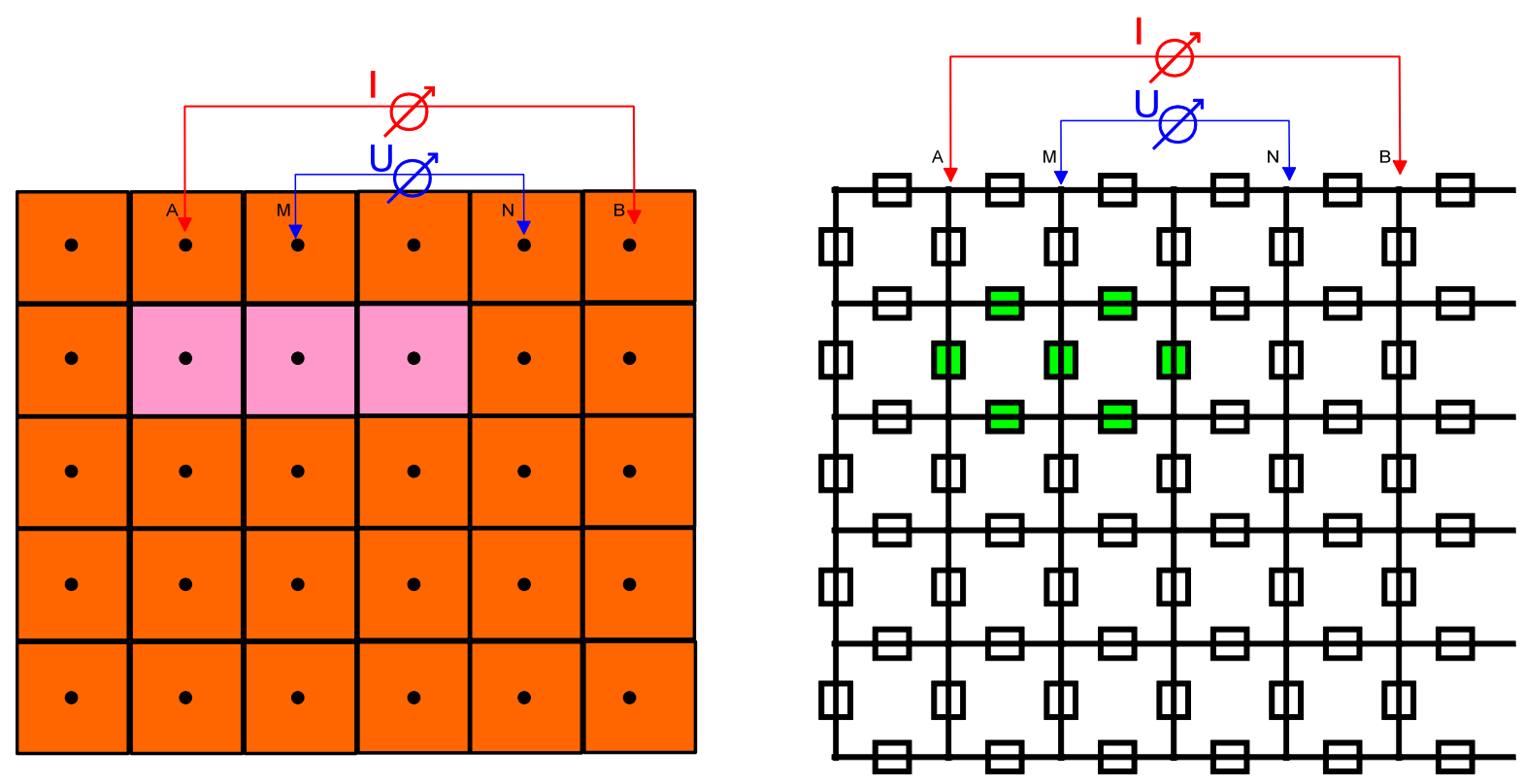

Fig. 3 Comparison of 2D model with distribution of blocks with given resistivity (in $\Omega \mathrm{m}$ ) and resistor network model formed by resistances of different resistors (in $\Omega$ ). Both models are completed with scheme of electrode arrays.

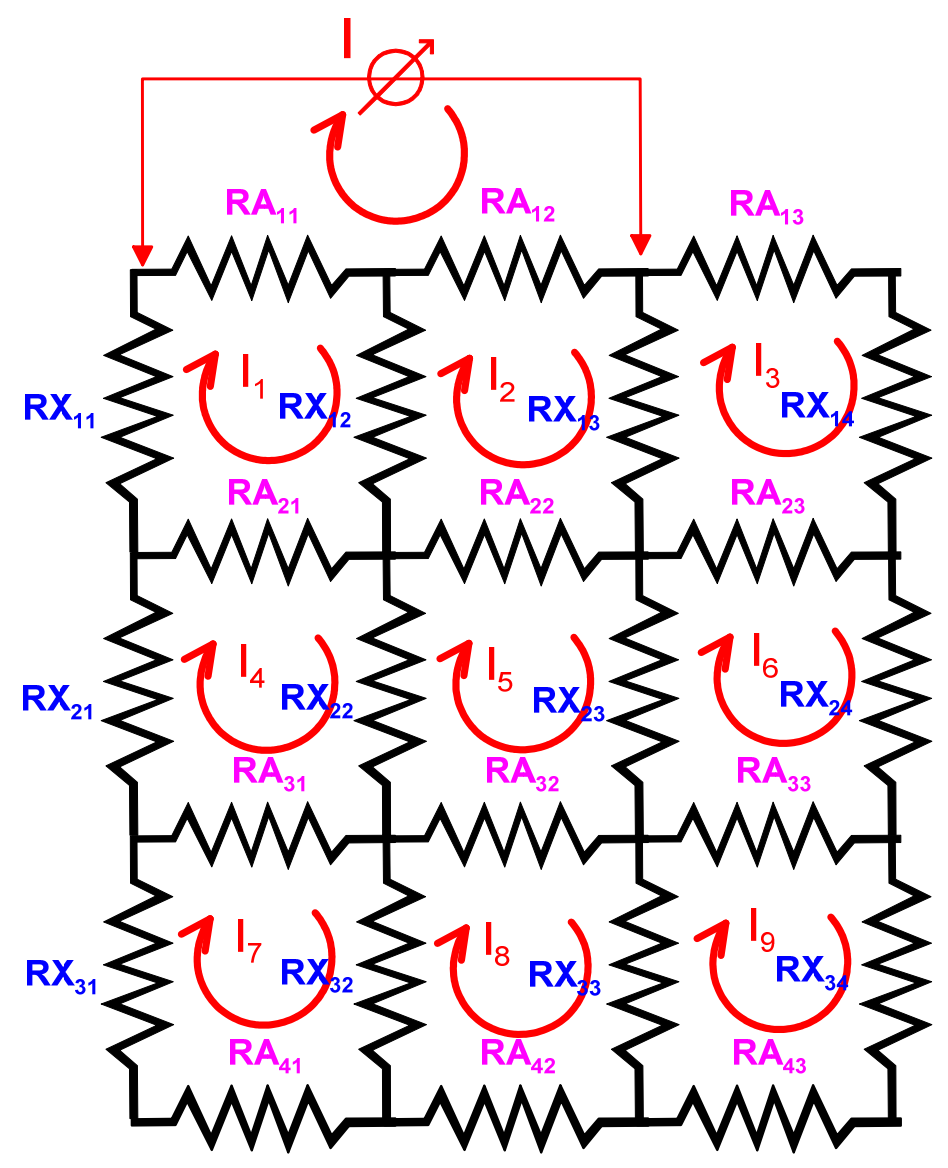

Fig. 4 Resistor network model with horizontal and vertical resistors. The red arrows denote loop currents. 


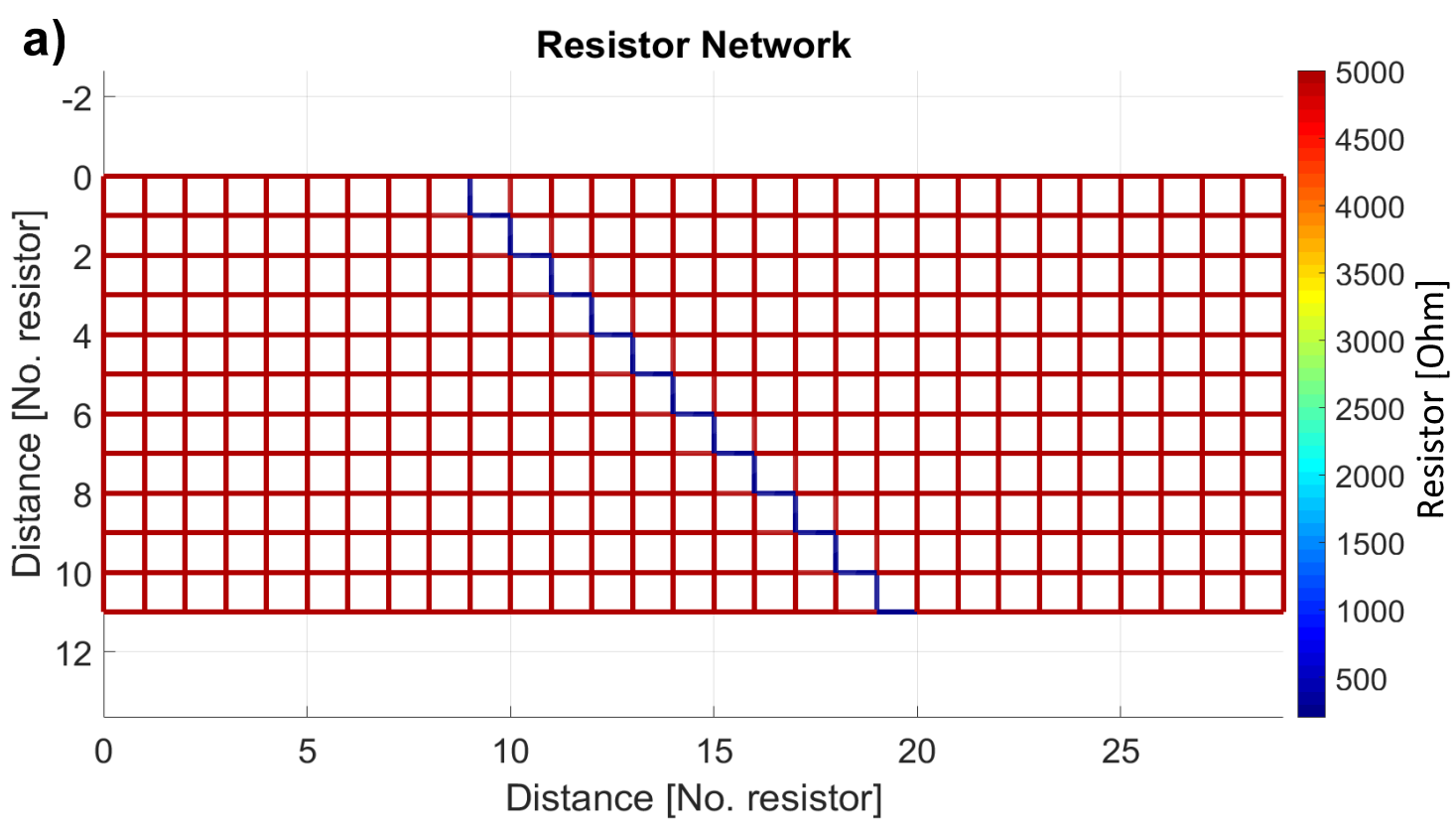

b)

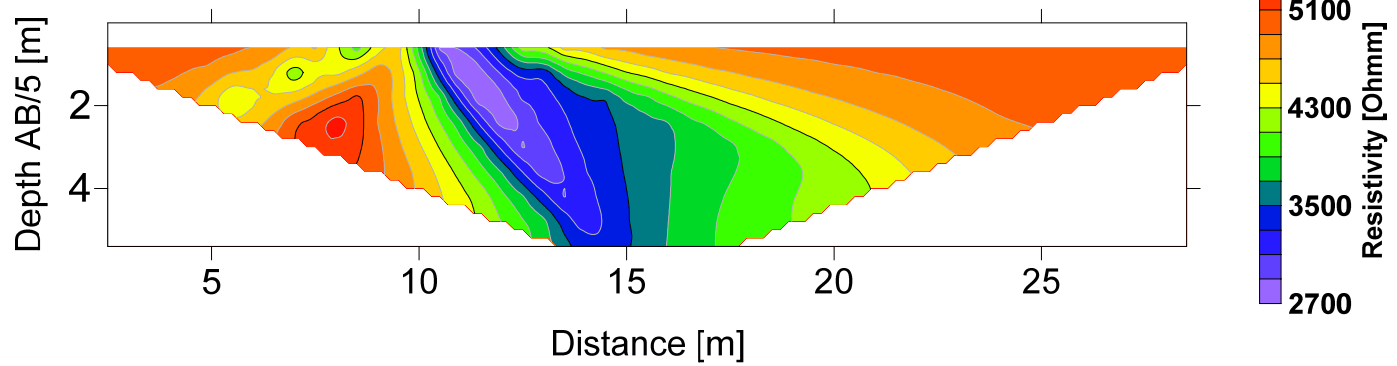

Fig. 5 Thin conductive fracture:

a) resistor network model, b) resistivity pseudo-section.

making the electric circuits one can use an equivalent circuit to replace selected resistor by several other resistors while its final resistance will equal to the original one. Such a property of the electric circuits proves a general principal ambiguity of the inverse problem's solution. However, we can set basic geometrical assumptions about the resistor network or consider the resistor network analogical to the blocklike environment's model with set resistivity values. As an example we can state an assumption of a resistor network that has geometrical dimensions of the side of $1 \mathrm{~m}$. In that case the network will behave analogically as the resistivity distribution model of $1 \times 1 \mathrm{~m}$ square blocks. A thin conductive fracture may be modelled via resistors with low resistance value. Then the necessity of simultaneous solution of the fracture dimension and its resistivity vanishes - it is only necessary to set an adequately low resistance value matching the measurement with an electrode connected directly into the fracture.

As an example of the apparent resistivity pseudo-cross section calculated via resistor network one can show an inclined conductive fracture model. For the sake of simplicity the size of the resistor network's mesh was chosen as $1 \mathrm{~m}$ (side's length). Surrounding non-conductive environment is modeled via $5000 \Omega$ resistors of 1-meter length, the conductive fracture is made by the $500 \Omega$ resistors. Considering the chosen 1-meter size of the resistors such a model can be compared to the model that would consist of $5000 \Omega \mathrm{m}$ and $500 \Omega \mathrm{m}$ resistivity blocks. Chosen meshes model and resulting apparent resistivity crosssection is in Figure 5.

\section{BUKOV UNDERGROUND LABORATORY - AN EXAMPLE OF MODEL CALCULATION}

As an example of the resistor network model's functionality we state a simulated apparent resistivity cross-section calculation corresponding to the measurement on the mine gallery's wall in the Bukov underground laboratory that works as an experimental analogue and test site for the future nuclear waste geological repository. The electrical resistivity tomography (ERT) measurement was carried out directly on the wall. The electrodes spacing was $50 \mathrm{~cm}$ with 38 electrodes in total. While proposing the model we also took in account the steel floor reinforcing rebar working as a deeper conductive medium during the measurement. Figure 6 shows relatively decent match between measured apparent resistivity cross-section and the calculated one made by using the resistor network model. 
a)

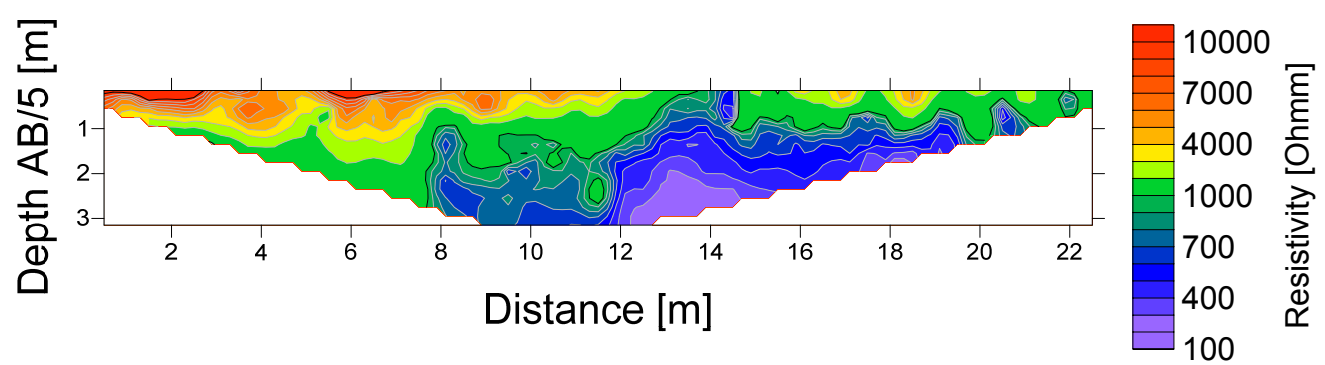

b)
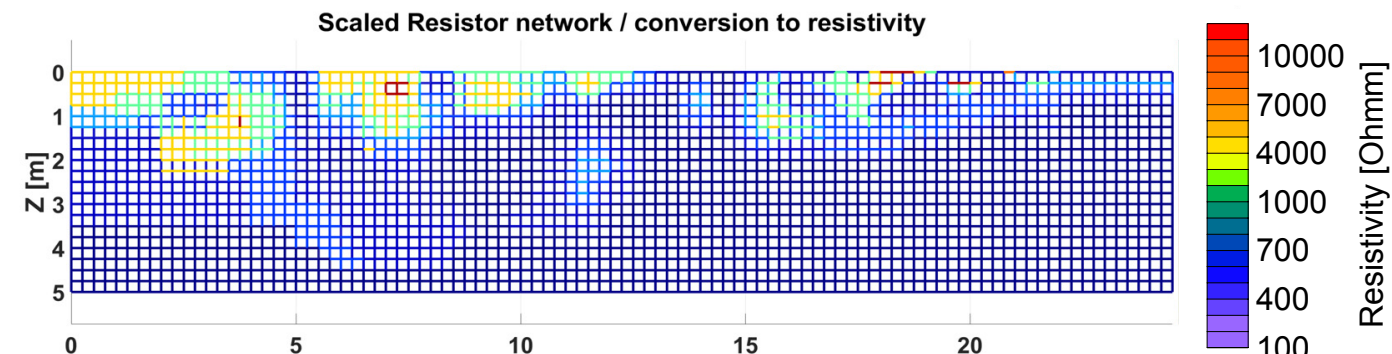

c)
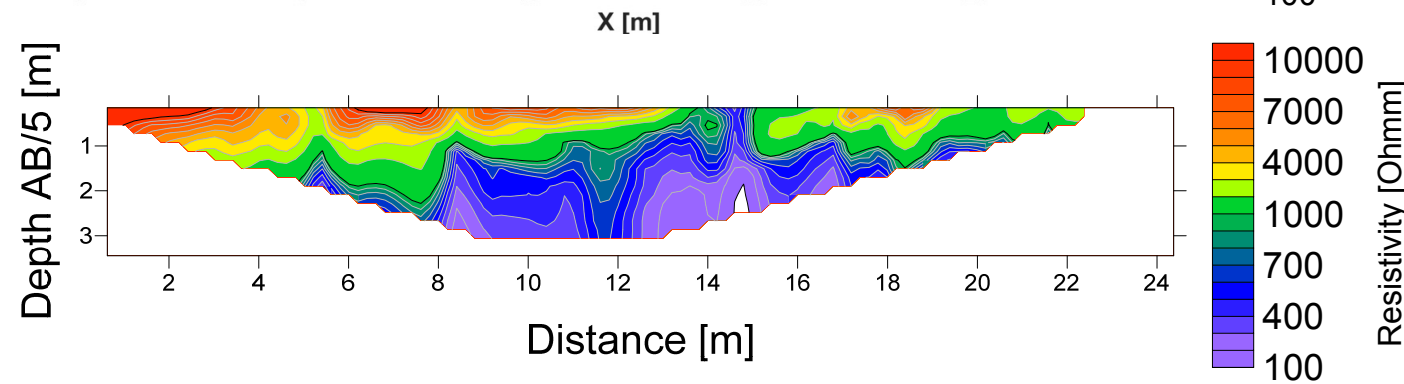

Fig. 6 Resistivity data and resistivity model from the Bukov underground laboratory.

a) Measured apparent resistivity section. b) Resistor network model.

c) Apparent resistivity section calculated for the resistor network model.

In Figure 7 we show the result of the Res2Dinv inversion made of the same data. The iteration process had to be carried out twice to cut down the RMS error of the inversion (via elimination of the poor data due to high contact resistance) to current 13.7 percent at the $4^{\text {th }}$ iteration. One can see that the inverted picture of the environment is generally similar but represents several differences - absolute values of the resistivities are generally higher for the surface zones as such values tend to increase with every iteration step. The inverted picture also distinguishes the subsurface conductive zone into two parts conductive narrow blue zone (first hundreds $\Omega \mathrm{m}$ ) and semi-conductive neighboring zone of around $1000 \Omega \mathrm{m}$ that was not distinguished in Figure 6 .

\section{DISCUSSION OF RESULTS}

The presence of a fracture in the geological environment can be a crucial issue. Among major task then belong - set the position and slope of the fracture, quantify the volume of it and guess on the physical parameters of the fracture, mainly resistivity in our case and eventually causes of such parameters' changes. The influence of the water saturation may be extremely strong as proven in our laboratory tests difference between partly and completely saturated fracture can vary in order-ranges. It was proven that solving these types of issues of strongly anisotropic environment is generally challenging for direct current methods. Using the resistor networks might increase the level of a detail we can get from such measurements like geometry of the fracture or its physical picture. Such an approach may be useful even in case of a geophysical long-term monitoring when narrowing down the rate of change is very important too.

An inverse problem is always ambiguous and automatically generated inversion is not always sufficient. This is caused by the fact that the inverse problem usually includes searching for a model of resistivity distribution which would generate an identical apparent resistivity distribution that would be measured. To narrow down principal ambiguity of resistivity methods, we can work with simplified models such as vertical electrical sounding (VES) where we are trying to approximate the geological medium with a 1D layered model. Even in this case, this includes the ambiguity caused by the equivalence principle. Solving more complex 2D or 3D forward geological problems is usually carried out via calculation of apparent resistivity values by the methods of finite-differences or finite-elements like in case of the Res2Dmod program. We proved that using the resistor network approach may be more reliable when expecting strong changes in measured environment. In such a case the finite-differences approach may be less-sufficient in getting rid of the ambiguity constraints. 


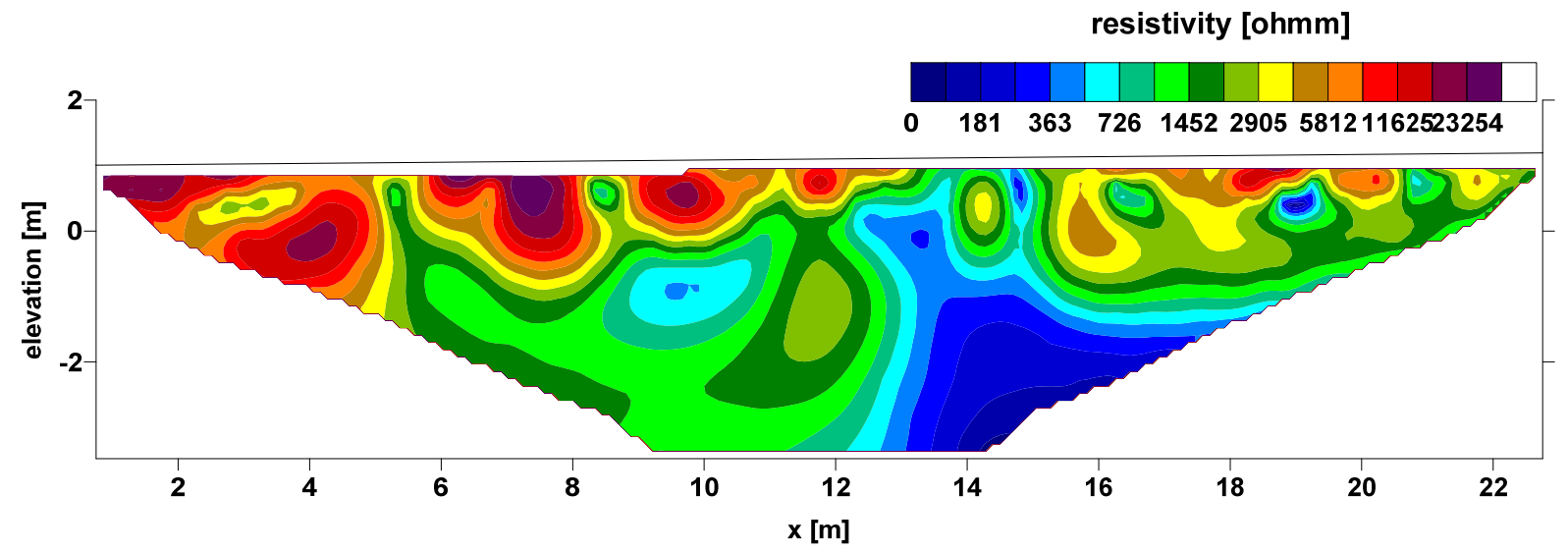

Fig. 7 Res2Dinv inverted cross-section from the Bukov underground laboratory.

The calculation of an apparent resistivity crosssection was solved only as a forward problem. However, every forward problem may have its inverse problem. If we were able to prepare an adequate inverse problem to the resistor network's model it might work better than in case of finite differences. This might be caused by more contrast and less smoothened cross-section of resistor network comparing to one of the Res2Dmod. A thin conductive fracture's manifestation is localized far more precisely. This will make the inverse problem better conditioned and more stable.

\section{CONCLUSIONS}

In this paper we describe a tool that was made for modeling the electrical resistivity forward problem. It was proven that the resistor network can be associated with geometrical dimensions and performs calculations of 2-D subsurface resistivity models. The resistor network uses generated crosssections similarly to Res2Dmod's approach but seems being more stable and detailed in some of the environments. Using the resistor network, we created a program that is equivalent to the finite-differences approach but allows working even with special cases like high-contrast fracture modeling. This can be useful in solving difficult applied problems such as in engineering geology or in geotechnics - our approach works more stable and reliable in case of very thin conductors, high resistivity contrasts or in strongly anisotropic environment. The resistor network may be promising when using data measured directly on the rock tunnel surface (repositories, galleries etc.).

\section{ACKNOWLEDGMENT}

The research was supported by the project CzechGeo/EPOS-Sci (CZ.02.1.01/0.0/0.0/16 013/0001800). The field data was gathered within the Radioactive waste repository authority (RAWRA) project "Longterm monitoring of the rock massif in the URF Bukov via non-destructive geophysical methods."

\section{REFERENCES}

Butler, S.L.: 2017, Analysis of the moments of the sensitivity function for resistivity over a homogeneous half-space: Rules of thumb for pseudoposition, offline sensitivity and resolution. J. Appl. Geophys., 143, 149-155. DOI: 10.1016/j.jappgeo.2017.06.005

ČSN EN 1936: 2007, Natural stone test methods Determination of real density and apparent density, and of total and open porosity. Standard is the Czech version of the European Standard EN 1936:2006.

Dahlin, T. and Loke, M.H.: 2018, Underwater ERT surveying in water with resistivity layering with example of application to site investigation for a rock tunnel in central Stockholm. Near Surf. Geophys., 16, 3, 230-237. DOI: 10.3997/1873-0604.2018007

Loke, M.H.: 2019, Tutorial: RES2DINVx64 ver. 4.09.

Norton, D. and Knapp, R.: 1977, Transport phenomena in hydrothermal systems; the nature of porosity. Am. J. Sci., 277, 913-936. DOI: 10.2475/ajs.277.8.913

Tullborg, E.L. and Larson, S.A..: 2006, Porosity in crystalline rocks - A matter of scale. Eng. Geol., 84, 1, 75-83. DOI: 10.1016/j.enggeo.2005.12.001

Reuschlé, T., Gbaguidi Haore, S. and Darot, M.: 2006, The effect of heating on the microstructural evolution of La Peyratte granite deduced from acoustic velocity measurements. Earth Planet. Sci. Lett., 243, 692-700. DOI: $10.1016 /$ j.epsl.2006.01.038

Ronczka, M., Voss, T. and Günther, T.: 2015, Cost-efficient imaging and monitoring of saltwater in a shallow aquifer by using long electrode ERT. J. Appl. Geophys., 122, 202-209.

DOI: 10.1016/j.jappgeo.2015.08.014

Rucker, D.F.: 2012, Enhanced resolution for long electrode ERT. Geophys. J. Int., 191, 1, 101-111. DOI: $10.1111 / \mathrm{j} .1365-246 X .2012 .05643 . \mathrm{x}$

Rukavičková, L.: 2008, Hydraulic conductivity and fracture frequency dependence in granite massifs. Geoscience Research Reports for 2007, 41, Czech Geological Survey, Prague, 232-235, (in Czech).

Vilhelm, J. and Jirků, J.: 2018, Forward modeling by means of resistor network - new tool for fracture detection in crystalline rocks. Geophysical Research Abstracts, 20, EGU2018-10381, EGU General Assembly.

Zeng, C., Wu, C. and Deng, W.: 2019, Thermal mechanical analysis of porous granite asperities in contact using probabilistic damage model. Int. J. Rock Mech. Min. Sci., 124, 104130.

DOI: $10.1016 /$ j.ijrmms.2019.104130 